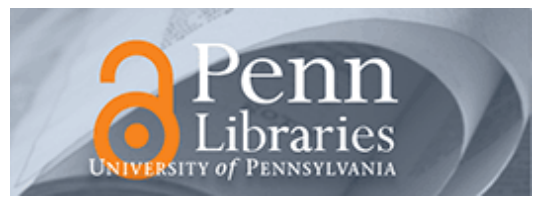

University of Pennsylvania

ScholarlyCommons

July 1998

\title{
Numerical investigation of the stabilization of the no-motion state of a fluid layer heated from below and cooled from above
}

Jie Tang

Aeroquip Corporation

Haim H. Bau

University of Pennsylvania, bau@seas.upenn.edu

Follow this and additional works at: https://repository.upenn.edu/meam_papers

\section{Recommended Citation}

Tang, Jie and Bau, Haim H., "Numerical investigation of the stabilization of the no-motion state of a fluid layer heated from below and cooled from above" (1998). Departmental Papers (MEAM). 113.

https://repository.upenn.edu/meam_papers/113

Copyright (1998) American Institute of Physics. This article may be downloaded for personal use only. Any other use requires prior permission of the author and the American Institute of Physics. Reprinted in Physics of Fluids, Volume 10, Issue 7, July 1998, pages 1597-1610.

Publisher URL: http://dx.doi.org/10.1063/1.869679

This paper is posted at ScholarlyCommons. https://repository.upenn.edu/meam_papers/113

For more information, please contact repository@pobox.upenn.edu. 


\title{
Numerical investigation of the stabilization of the no-motion state of a fluid layer heated from below and cooled from above
}

\begin{abstract}
The feasibility of controlling flow patterns of Rayleigh-Bénard convection in a fluid layer confined in a circular cylinder heated from below and cooled from above (the Rayleigh-Bénard problem) is investigated numerically. It is demonstrated that, through the use of feedback control, it is possible to stabilize the nomotion (conductive) state, thereby postponing the transition from a no-motion state to cellular convection. The control system utilizes multiple sensors and actuators. The actuators consist of individually controlled heaters positioned on the bottom surface of the cylinder. The sensors are installed at the fluid's midheight. The sensors monitor the deviation of the fluid's temperatures from preset desired values and direct the actuators to act in such a way so as to eliminate these deviations. The numerical predictions are critically compared with experimental observations.
\end{abstract}

\section{Keywords}

Rayleigh-Benard instability, Benard convection, feedback, flow control, sensors, actuators

\section{Comments}

Copyright (1998) American Institute of Physics. This article may be downloaded for personal use only. Any other use requires prior permission of the author and the American Institute of Physics. Reprinted in Physics of Fluids, Volume 10, Issue 7, July 1998, pages 1597-1610.

Publisher URL: http://dx.doi.org/10.1063/1.869679 


\title{
Numerical investigation of the stabilization of the no-motion state of a fluid layer heated from below and cooled from above
}

\author{
Jie Tang \\ Corporate Technology Laboratory, Aeroquip Corporation, Ann Arbor, Michigan 48105-1530 \\ Haim H. Bau ${ }^{\text {a) }}$ \\ Department of Mechanical Engineering and Applied Mechanics, University of Pennsylvania, Philadelphia, \\ Pennsylvania 19104-6315
}

(Received 13 May 1997; accepted 24 March 1998)

\begin{abstract}
The feasibility of controlling flow patterns of Rayleigh-Bénard convection in a fluid layer confined in a circular cylinder heated from below and cooled from above (the Rayleigh-Bénard problem) is investigated numerically. It is demonstrated that, through the use of feedback control, it is possible to stabilize the no-motion (conductive) state, thereby postponing the transition from a no-motion state to cellular convection. The control system utilizes multiple sensors and actuators. The actuators consist of individually controlled heaters positioned on the bottom surface of the cylinder. The sensors are installed at the fluid's midheight. The sensors monitor the deviation of the fluid's temperatures from preset desired values and direct the actuators to act in such a way so as to eliminate these deviations. The numerical predictions are critically compared with experimental observations. () 1998 American Institute of Physics. [S1070-6631(98)01007-1]
\end{abstract}

\section{INTRODUCTION}

The ability to control complex convective flow patterns is important in both technology and fundamental science. In many technological processes, the naturally occurring flow patterns may not be the optimal ones. By controlling the flow, one may be able to optimize the process. The ability to stabilize otherwise nonstable states may also assist one in gaining deeper insights into the dynamics of flows. Since fluid flow phenomena are highly nonlinear and possess many degrees of freedom, the flow control problem is far from trivial.

In prior experimental and theoretical investigations, ${ }^{1-5}$ various linear and nonlinear control strategies were used to alter the bifurcation structure of the convective motion in a thermal convection loop heated from below and cooled from above. For example, with the aid of a controller, the transition from a no-motion to a motion state was delayed, naturally occurring chaotic motion in the loop was suppressed, otherwise nonstable periodic orbits embedded in the chaotic attractor were stabilized, subcritical bifurcations were rendered supercritical, and chaos was induced under conditions in which the flow normally would be laminar. In the case of the thermal convection loop, temporally complex flows were controlled. The objective of this paper is to determine whether systems with many spatial degrees of freedom can also be successfully controlled. To this end, we investigate the feasibility of delaying the transition from the no-motion (pure conduction) to the motion state in the RayleighBénard problem of a fluid layer heated from below and cooled from above. We have chosen this problem for study

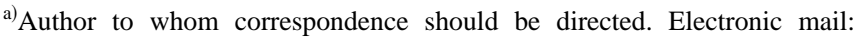
bau@seas.upenn.edu
}

because the uncontrolled problem has been extensively studied and the phenomena observed are relatively well understood. The Rayleigh-Bénard problem is also of technological importance since it serves as a paradigm for many material processing applications.

A considerable amount of work has been devoted to delaying the onset of Rayleigh-Bénard convection. Most of these attempts included the use of predetermined (open loop control), time-periodic modulation of the temperature difference across the layer (for lucid reviews, see Davis, ${ }^{6}$ and Donnelly $^{7}$ ). Unfortunately, this technique provides only marginal stabilization. Moreover, periodic modulation may lead to a subcritical bifurcation, ${ }^{8}$ thereby causing the no-motion state to be stable only for small disturbances. Kelly ${ }^{9}$ and Kelly and $\mathrm{Hu}^{10}$ proposed delaying the onset of cellular convection by causing the fluid in the layer to oscillate slowly about a zero mean with out-of-phase, two horizontal velocity components. In contrast, our objective is to maintain a state of complete rest. While Kelly's controller operates in an "open loop" mode, our controller is a closed loop (feedback) controller.

In theoretical studies focusing on an infinite layer of Newtonian and Darcian fluids with various heating conditions, Tang ${ }^{11}$ and Tang and $\mathrm{Bau}^{12-15}$ have shown that with the aid of a feedback controller, the critical Rayleigh number for the onset of convection can be significantly increased. Using the control strategies of Tang and Bau, Howle, ${ }^{16-18}$ and Tang and $\mathrm{Bau}^{19}$ demonstrated experimentally that feedback control can be used to reduce the intensity of twodimensional convection in a slender box and in an upright circular cylinder, respectively. It is interesting to note that similar control strategies can be used to modify the flow patterns of shear flows ${ }^{20}$ and surface tension driven flows. ${ }^{21}$ In this investigation, we study theoretically the effect of the 
controller on Rayleigh-Bénard convection in an upright circular cylinder. To the extent possible, the theoretical results are compared with experimental data. Our objective is to enhance our understanding of the flow patterns in the presence and absence of the controller, to study the effect of a finite number of sensors and actuators on a controller's performance, and eventually to use the numerical code to optimize the controller.

\section{THE MATHEMATICAL MODEL AND THE NUMERICAL CODE}

We consider an upright circular cylinder of radius (a) and height $(H)$ filled with a fluid, heated from below and cooled from above. Two different heating conditions are considered. In one case (A), the nominal bottom temperature is specified. In the second case (B), the nominal bottom heat flux is specified. In the absence of a controller, either the bottom temperature (case A) or the bottom heat flux (case B) are uniform. We use these two types of heating conditions because later on, we will compare our numerical predictions with experimental observations. In our experiments, we used an array of heaters mounted on a silicon wafer and the true heating condition was somewhere between the uniform temperature (case A) and uniform flux (case B) condition.

The mathematical model consists of the OberbeckBoussinesq equations ${ }^{22}$ formulated in cylindrical coordinates. The cylinder's height, $H$, is the length scale; the velocity $U=(\alpha / H) \sqrt{R \operatorname{Pr}}$ is the velocity scale; $\mu U / H$ is the pressure scale; and $H / U$ is the time scale. $\alpha$ is the fluid's thermal diffusivity; $\mu$ is the shear viscosity; $\nu$ is the kinematic viscosity; $\operatorname{Pr}=\nu / \alpha$ is the fluid's Prandtl number; $\beta$ is the thermal expansion coefficient; $g$ is the gravitational acceleration; and $\lambda_{f}$ is the thermal conductivity of the fluid. In case $\mathrm{A}$, the nominal temperature difference, $\Delta T_{T B}$ between bottom and top, is the temperature scale and the $\Delta T_{T B}$-based Rayleigh number is $R_{T}=g \beta \Delta T_{T B} H^{3} / \alpha \nu$. In case $\mathrm{B}, q H / \lambda_{f}$ is the temperature scale and the heat-flux based Rayleigh number is $R_{q}=g \beta q H^{4} / \lambda_{f} \alpha \nu$, where $q$ is the nominal heat flux. The aspect ratio of the cylinder $(\gamma=\mathbf{a} / H)$ is the ratio of the cylinder's radius and height. The nondimensional temperature is denoted $\theta(r, \varphi, z, t)$, where $0 \leqslant r \leqslant \gamma$, $0 \leqslant \varphi \leqslant 2 \pi, 0 \leqslant z \leqslant 1$, and $t \geqslant 0$ are, respectively, the cylindrical coordinates and time. $u, v$, and $w$ denote, respectively, the radial, angular, and vertical velocity components. The critical Rayleigh number for the onset of convection is denoted by $R_{x, c}$, where $x$ stands for either $T$ or $q$. The reduced Rayleigh number $(\chi)$ is the ratio between the actual and the critical Rayleigh numbers, $\chi=R_{x} / R_{x, c}$.

For the purposes of code verification, we occasionally used slip boundary conditions at some of the solid boundaries. When we carried out the actual numerical simulations, nonslip boundary conditions were specified at all solid boundaries.

The side wall thermal boundary condition presented a problem. Our experimental apparatus was constructed from Plexiglas and the working fluid was silicon oil. The thermal conductivity of the Plexiglas wall was slightly higher than that of the silicon oil. Thus the side wall participated in the heat transfer process. In order to minimize computer time and to avoid the need of solving a conjugate heat transfer problem, we did not compute the temperature distribution in the side wall.

A simplified, crude model of the side wall thermal boundary condition was provided by Buell and $\mathrm{Catton}^{23}$

$$
\left(\frac{\partial^{2}}{\partial z^{2}}+\frac{1}{\gamma} \frac{\partial^{2}}{\partial \varphi^{2}}\right) \theta(\gamma, \varphi, z)=\frac{\Lambda}{\gamma} \frac{\partial \theta(\gamma, \varphi, z)}{\partial r}
$$

where $\Lambda=\lambda_{f} \mathbf{a} /\left(\lambda_{w} t_{w}\right), \lambda_{w}$ is the thermal conductivity of the side wall material, and $t_{w}$ is the side wall's thickness. When $\Lambda \rightarrow \infty$, the wall is perfectly insulating. When $\Lambda=0$, the wall is conducting and its temperature distribution is independent of the processes occurring in the fluid. In our experiments, ${ }^{11,19} \Lambda \sim 0.35$. Thus the experimental data is expected to fall between the insulating and conducting cases.

In our numerical simulations, we considered the two limiting cases: insulated side wall $(\Lambda \rightarrow \infty$, case $C)$ or perfectly conducting side wall $(\Lambda=0$, case $D)$. To clarify our notation, when we refer to case $\mathrm{AC}$, we mean to say that the bottom temperature is uniform (case A) and the side wall is insulated (case C).

We used a staggered control volume approach to discretize the equations. The momentum and energy equations were integrated over a control volume, resulting in algebraic equations which were subsequently solved using the SIMPLER algorithm. ${ }^{24} \mathrm{We}$ denote the number of grid points in the $(r, \varphi, z)$ directions by $\left(n_{r}, n_{\varphi}, n_{z}\right)$. Backward differences were used to approximate the time derivatives. The convective terms were approximated using the QUICK scheme ${ }^{25}$ with a modified formulation next to solid boundaries. ${ }^{26}$ Special care was given to the singularity at the cylinder's center, $r=0$, where the radial and angular components of the velocity are not defined. By constructing a small circle of radius $\varepsilon$ $(\varepsilon \rightarrow 0)$ around the center, we obtained the conditions: $u(\varepsilon, \varphi, z)=-u(\varepsilon, \varphi+\pi, z) \quad$ and $\quad \partial u(\varepsilon, \varphi, z) / \partial r=\partial u(\varepsilon, \varphi$ $+\pi, z) / \partial r$ for the radial component of the velocity. Similar relationships were obtained for the angular component of the velocity. We mention these conditions at $r=0$ since many researchers have erroneously prescribed zero radial velocity next to $r=0$.

The discretized equations were solved using a projection-based, iterative solver, GMRES (Generalized Minimum Residual Method) with preconditioning. ${ }^{27}$ This solver proved to be more robust and somewhat more efficient (on average about $11 \%$ faster) than the Tri-diagonal Matrix Algorithm (TDMA) with Alternative Direction Implicit Iterations (ADI), which we initially tried. The iterations were terminated when the residues of the momentum, energy, and mass conservation equations were all smaller than $10^{-12}$. We also verified mass conservation by computing the mass flow through various vertical and horizontal cross sections of the cylinder and on vertical circular surfaces and establishing that the net mass flow was smaller than $10^{-12}$. Finally, we verified that in case $\mathrm{C}$, under time-independent conditions, the differences in the heat flow through any horizontal cross section were also smaller than $10^{-12}$. 


\section{CODE VERIFICATION}

The code was verified by assuring that conservation laws were satisfied and verifying that the results were reasonably grid size independent as well as by comparing our results with those of other researchers and, when feasible, with analytic solutions. For axisymmetric flow of a $\operatorname{Pr}=6.7$ fluid in a cylinder with an aspect ratio (radius/height) of $\gamma=1.75$, type AC thermal boundary conditions, slip walls, and reduced Rayleigh numbers, $\chi \leqslant 2$, our computed Nusselt numbers (with $15 \times 4 \times 15$ grid points) agreed within $0.3 \%$ with the two-dimensional, axisymmetric calculations of Jones, Moore, and Weiss, ${ }^{28}$ who utilized $48 \times 84$ grid points. For cylinders with aspect ratios $\gamma=1.67\left(n_{r}=48, n_{z}=80\right)$ and $\gamma=1.42\left(n_{r}=48, n_{z}=80\right)$, our results $\left(n_{r}=21, n_{\varphi}=4\right.$, $n_{z}=21$ ) agreed with those of Jones, Moore, and Weiss within $0.7 \%$ and $4.5 \%$, respectively. Additional comparisons with three-dimensional computations of other researchers are provided later in this paper.

In a cylinder of aspect ratio $\gamma=0.5, \operatorname{Pr}=0.7$, type $\mathrm{BC}$ thermal boundary conditions, nonslip bottom and top, and slip side wall, we used our code to compute the critical Rayleigh number at the onset of convection and compared the results with the analytical ones (the Appendix). The numerically computed critical Rayleigh number, $R_{q, c}$, was obtained by observing whether intentionally introduced disturbances amplify or decay. We started the computations by specifying the Rayleigh number, a conductive temperature field, and a small disturbance. We assumed that close to criticality, the disturbances grew/decayed as exponential functions of time. Accordingly, on a log-log plot, we depicted one of the variables, i.e., the vertical velocity component at a midheight point, as a function of time. By fitting a straight line to the data, we obtained an estimate of the growth rate, $\sigma$. The computations were repeated for a few subcritical and supercritical Rayleigh numbers and the various growth rates were plotted as functions of the Rayleigh number. In the vicinity of the critical Rayleigh number, the growth rate, $\sigma$, varied nearly linearly as a function of the Rayleigh number, $R$. For

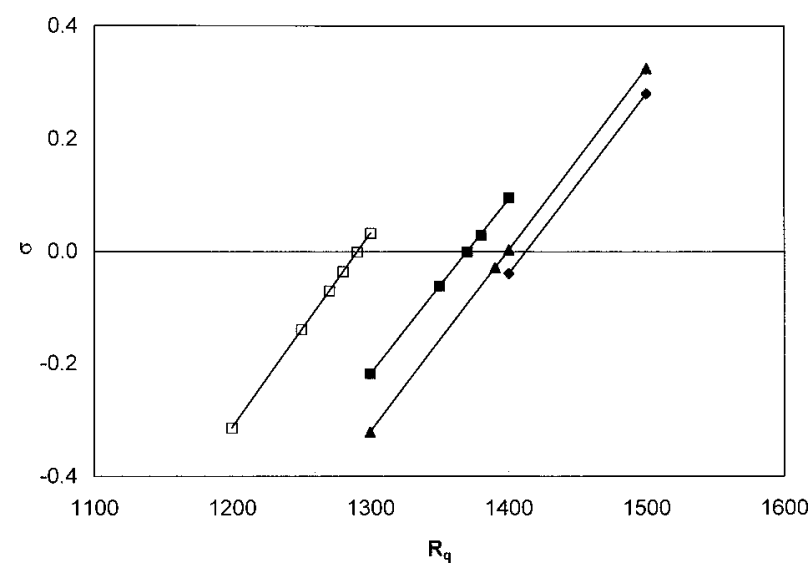

FIG. 1. The growth rate $\sigma$ is depicted as a function of the Rayleigh number, $R_{q}$, and the mesh size. Hollow squares, solid squares, triangles, and diamonds correspond, respectively, to mesh sizes $(7 \times 8 \times 7),(11 \times 18 \times 11)$, $(15 \times 20 \times 15)$, and $(19 \times 24 \times 19)$. The cylinder aspect ratio $\gamma=0.5$. $\operatorname{Pr}=0.7$. The thermal boundary conditions are of type BC and slip is allowed at the side wall. example, for a cylinder with an aspect ratio $\gamma=0.5$ and $\operatorname{Pr}=0.7$, Fig. 1 depicts the growth rate $\sigma$ as a function of the Rayleigh number, $R_{q}$, and the mesh size. Hollow squares, solid squares, triangles, and diamonds correspond, respectively, to mesh sizes $(7 \times 8 \times 7), \quad(11 \times 18 \times 11)$, $(15 \times 20 \times 15)$, and $(19 \times 24 \times 19)$. Unfortunately, due to computer power limitations, we were not able to further refine the grid. The estimate of the critical Rayleigh number which corresponds to a zero growth rate $(\sigma=0)$ was obtained by interpolation.

Figure 1 indicates that the critical number was not independent of the mesh size and that it increased as the mesh size was refined. To estimate the grid-independent $R_{q, c}$, we depicted the critical Rayleigh number as a function of $\left(n_{r} \times n_{\varphi} \times n_{z}\right)^{-1}$, extrapolated the curve to zero mesh size using a quadratic curve fit, and obtained the "grid sizeindependent" " critical Rayleigh number, $R_{q, c}=1420$. This estimate falls short by about $5.8 \%$ from the analytically obtained value of 1508 (the Appendix).

\section{THE UNCONTROLLED CASE}

We first carried out computations in the absence of a controller. The purpose of these numerical experiments was to provide a reference state against which the controlled flow would be compared. The results are also of interest all by themselves since they provide information on RayleighBénard convection in an upright circular cylinder. All the computations were carried out for nonslip walls and a cylinder of aspect ratio $\gamma=0.5$. In order to facilitate comparison with other researchers' results, computations were carried out for various Prandtl numbers. When we compared the computational results with our own experimental data, we specified $\operatorname{Pr}=135$, which is still much smaller than the $\operatorname{Pr} \sim 4.77 \times 10^{5}$ of the silicon fluid used in our experiments, but sufficiently large so that further increases in the Prandtl number were not likely to introduce significant changes in the simulations' results.

First, we computed the critical Rayleigh number for the onset of convection in a cylinder with AC type thermal boundary conditions. The method we used to obtain the critical Rayleigh number was described in Sec. III. Upon extrapolation, we obtained the grid-independent critical Rayleigh number $R_{T, c} \sim 3735 \pm 5$. Our predicted $R_{T, c}$ value is in reasonable agreement with results obtained by Buell and Catton $^{23}$ who used linear stability and a Galerkin technique to obtain $R_{T, c} \sim 3800$ and Neumann ${ }^{29}$ who used nonlinear, finite difference numerical simulations to obtain $R_{T, c} \sim 3610$. In contrast, using similar techniques to those of Buell and Catton, Charlson and $\mathrm{Sani}^{30}$ obtained $R_{T, c} \sim 4500$. This relatively high estimate of $R_{T, c}$ was apparently caused by inappropriate treatment of the singularity at $r=0$. We repeated the same calculations for thermal boundary conditions of type AD to obtain $R_{T, c} \sim 7,500 \pm 50$.

Next, we computed the temperature and flow field in the cylinder with AC type thermal boundary conditions. In order to facilitate comparison with other workers, the simulations were initially carried out for $\operatorname{Pr}=6.7$ fluid. When $R_{T}=17500, \chi \sim 4.7$, and $\operatorname{Pr}=6.7$, Figure 2 depicts the verti- 


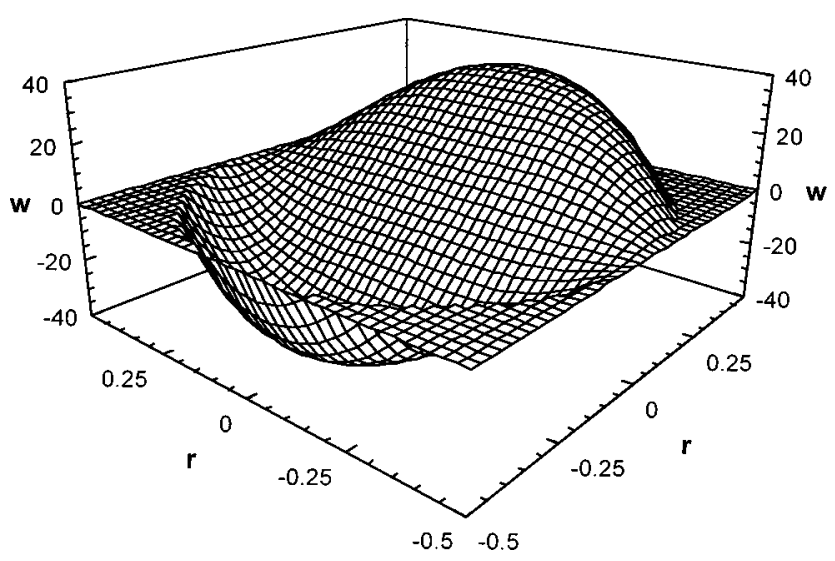

FIG. 2. The vertical component of the velocity, $w$, at the cylinder's midheight $(z=0.5)$ is depicted as a function of $r$ and $\varphi . \gamma=0.5 . R_{T}=17,500$. $\operatorname{Pr}=6.7$. Thermal boundary conditions are of type AC.
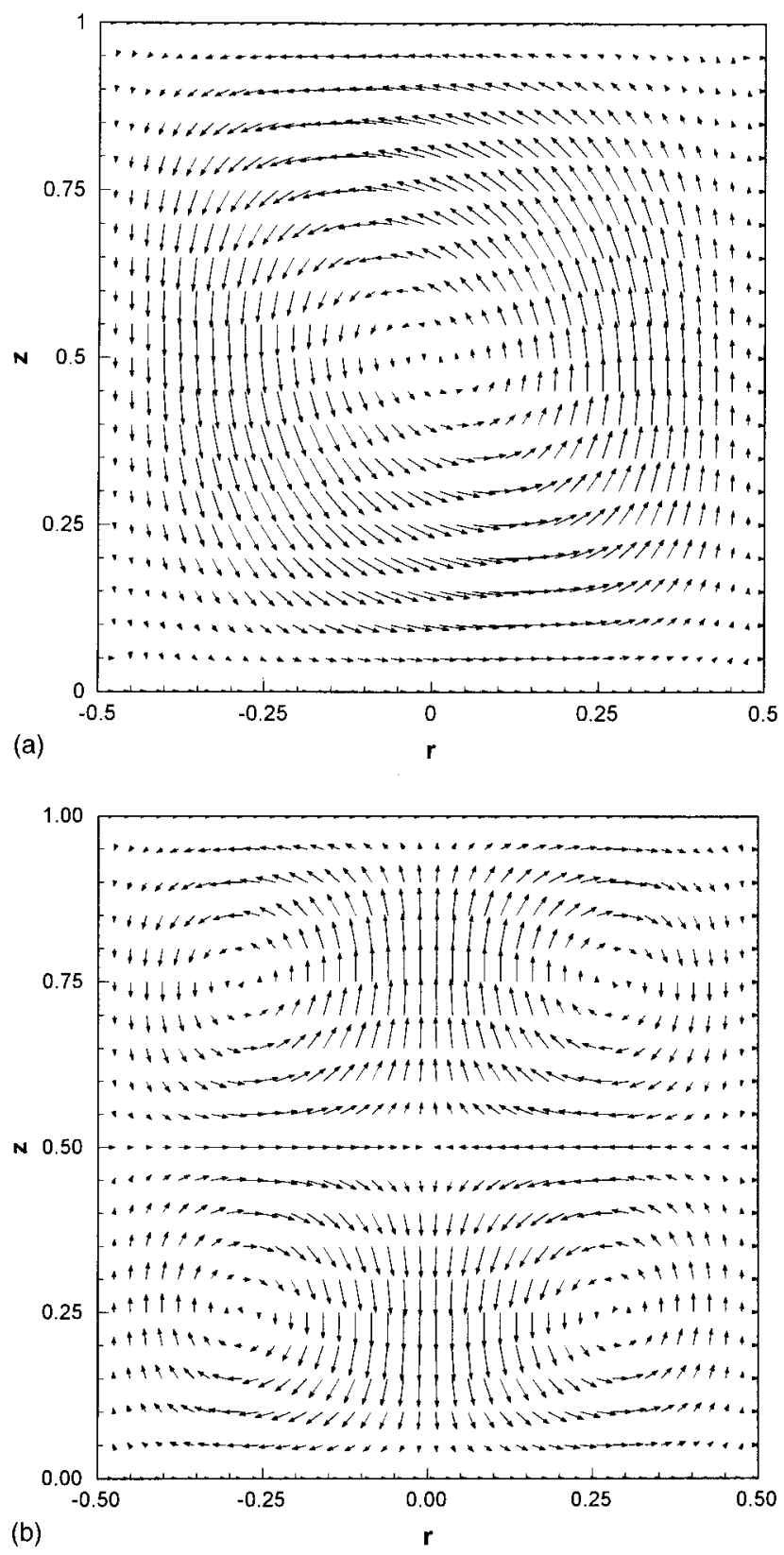

cal component of the velocity, $w$, at the cylinder's midheight $(z=0.5)$ as a function of $r$ and $\varphi$. Figure 2 illustrates that the postcritical flow field consists of a single convective cell. The flow is symmetric with respect to the plane $\varphi=\pi / 2$. The cylinder is divided into two parts by the vertical $\varphi=0$ plane. The flow ascends in the sector $\pi<\varphi<2 \pi$ and descends in the sector $0<\varphi<\pi$. This flow pattern is qualitatively similar to the predictions of linear theory ${ }^{23}$ and the experimental observations of Müller, Neumann, and Webber, ${ }^{31}$ Tang, ${ }^{11}$ and Tang and Bau. ${ }^{19}$ The results are also in good quantitative agreement with the numerical computations. ${ }^{29,32}$

Figures 3(a) and 3(b) depict, respectively, the projections of the vector fields on the plane of symmetry $(\varphi=\pi / 2)$ and the plane $(\varphi=0)$ when $R_{T}=17500, \operatorname{Pr}$ $=6.7$, and $\chi \sim 4.7$. Figure 3(c) depicts the isotherms in the $\varphi=\pi / 2$ plane. The flow field depicted in Fig. 3(a) and the isotherms depicted in Fig. 3(c) are consistent with a single

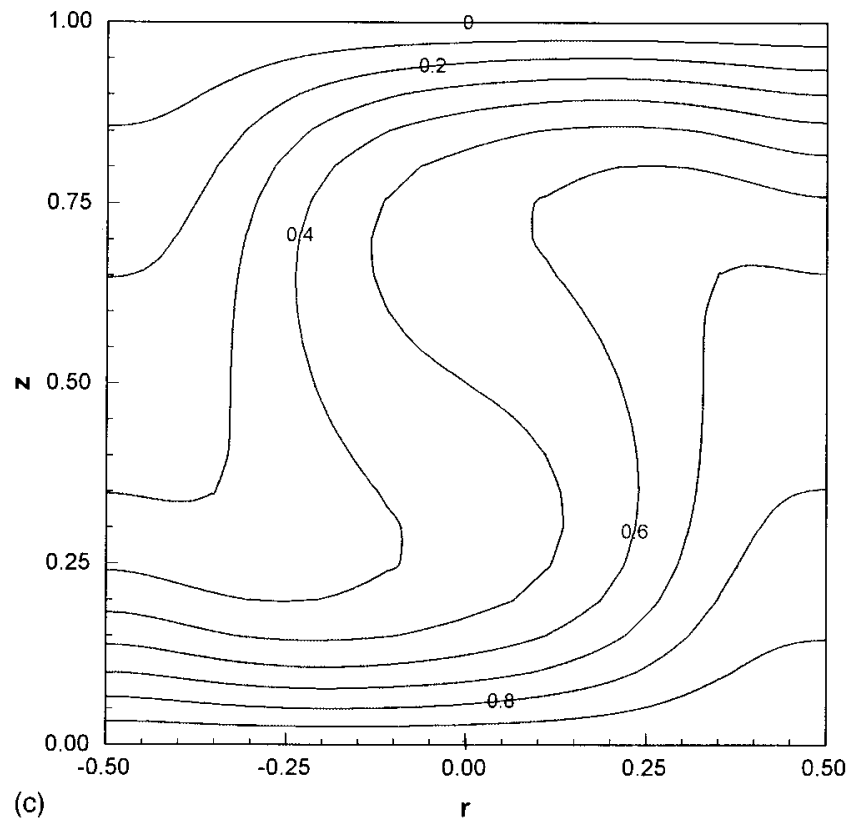

FIG. 3. The projections of the vector fields on the planes, $\varphi=\pi / 2$ (a) and $\varphi=0$ (b), and the isotherms in the plane $\varphi=\pi / 2$ (c) are depicted as functions of $r$ and $z$. The thermal boundary conditions are of type AC, $\chi$ $\sim 4.7, \operatorname{Pr}=6.7$ and $\gamma=0.5$. 


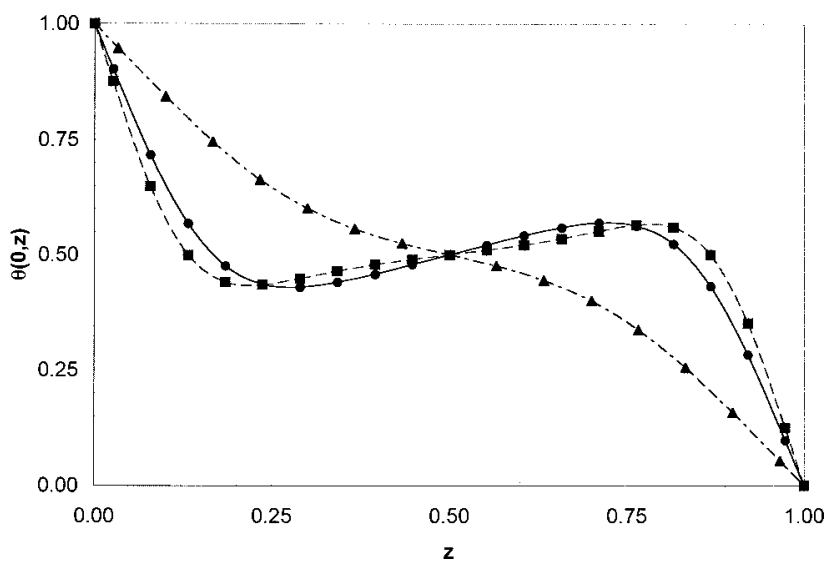

FIG. 4. The temperature along the cylinder axis, $\theta(0, z)$, is depicted as a function of the vertical coordinate $z$ when $R_{T}=5000$ ( $\chi \sim 1.3$, upright triangles), $R_{T}=17500$ ( $\chi \sim 4.7$, solid circles) and $R_{T}=50000$ ( $\chi \sim 13.4$, solid squares). $\operatorname{Pr}=6.7, \gamma=0.5$ and the thermal boundary conditions are of type AC.

cell flow pattern. The flow field in the $\varphi=0$ plane [Fig. 3(b)] exhibits four counter-rotating eddies.

Witness that the temperature field [Fig. 3(c)] exhibits a temperature inversion. This phenomenon is shown more clearly in Fig. 4, which depicts the temperature along the cylinder's axis $(r=0)$ as a function of the vertical coordinate $z$ for $R_{T}=5000(\chi \sim 1.3), R_{T}=17500(\chi \sim 4.7)$ and 50000 $(\chi \sim 13.4)$. At $z=0$, the nondimensional temperature is equal to 1 . When $R_{T}=5000$ (upright triangles), the temperature decreases monotonically as $z$ increases. This is no longer true for larger $R_{T}$ values. When $R_{T}=17500$ (solid circles), as $z$ increases, the temperature along the cylinder's axis initially decreases, attains a local minimum, then increases again to a local maximum, and finally declines to zero at the top boundary $(z=1)$. The magnitude of the temperature dip does not change much as the Rayleigh number further increases (i.e., $R_{T}=50000$, solid squares).

Figure 5 depicts the vertical components of the velocity

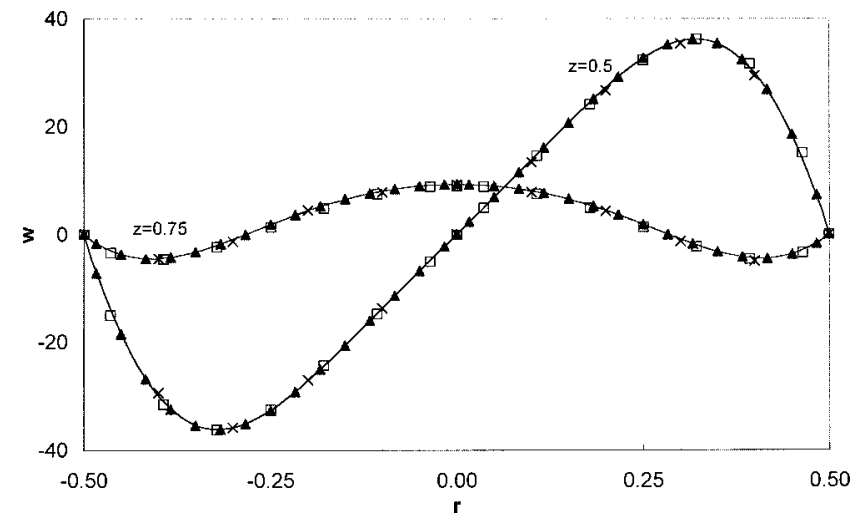

FIG. 5. The vertical components of the velocity at $z=0.5$ along the cylinder's diameter that lies in the symmetry plane $(\varphi=\pi / 2)$ and at $z=0.75$ along the diameter that lies in the plane $\varphi=0$ are depicted as functions of the radial coordinate, $r . R=17500, \chi \sim 4.7, \operatorname{Pr}=6.7$, and $\gamma=0.5$. The hollow squares, upright solid triangles, and solid lines represent, respectively, our own results with $(7 \times 8 \times 7),(15 \times 20 \times 15)$, and $(19 \times 24 \times 19)$ grid points. The $(x)$ represents data computed by Muller, Neumann, and Weber (1984, with $24 \times 24 \times 24$ mesh points).

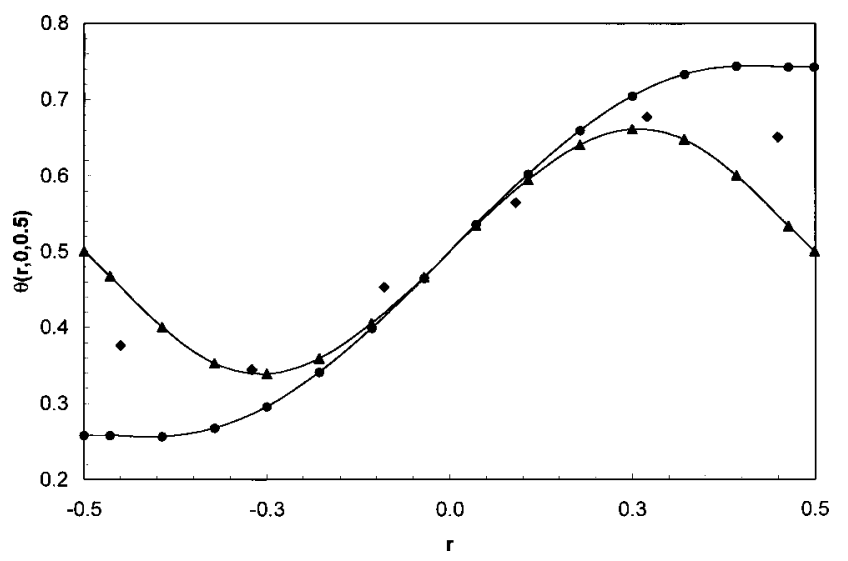

FIG. 6. The computed temperature when the boundary conditions are of type AC (solid circles) and type AD (solid triangles) and the nondimensional measured temperature (solid diamonds) are depicted as functions of the radius. $\chi=1.5, \operatorname{Pr}=135$, and $\gamma=0.5$. The profiles were chosen in such a way as to include the maximum and minimum midheight temperatures.

at $z=0.5$ along the cylinder's diameter that lies in the symmetry plane $(\varphi=\pi / 2)$ and at $z=0.75$ along the diameter that lies in the $\varphi=0$ plane. $R=17500$ and $\chi \sim 4$.7. The hollow squares, upright solid triangles, and solid lines represent, respectively, our own results with $(7 \times 8 \times 7),(15 \times 20 \times 15)$, and $(19 \times 24 \times 19)$ grid points. The $(x)$ represent data computed by Müller, Neumann, and Weber ${ }^{31}$ (with $24 \times 24 \times 24$ mesh points). Witness the good agreement between the various computational results. The velocity profile along the diameter at $z=0.5$ is consistent with up flow in one half of the cylinder and down flow in the other half. The velocity profile at $z=0.75$ is consistent with the four eddies seen in Fig. 3(b). Qualitatively similar results to those shown in Figs. 2-5 were obtained for the high Prandtl number cases.

In the experiments, we measured the temperature distribution at the fluid's midplane $(z=0.5)$. In Fig. 6, we compare the measured and computed temperature distributions along a diameter on which we observed the minimum and maximum temperatures. As a function of the radius, the figure depicts the nondimensional measured temperature (solid diamonds) and the computed temperatures using $\mathrm{AC}$ type (solid circles) and AD type (solid triangles) thermal boundary conditions. $\chi=1.5$ and $\operatorname{Pr}=135$. In the experiments, the nondimensional temperature is defined as $\theta=(T$ $\left.-T_{0}\right) / \Delta \bar{T}_{B T}$, where $T, T_{0}$, and $\Delta \bar{T}_{B T}$ are, respectively, the measured midheight temperature, the temperature of the top boundary, and the difference between the average bottom temperature and the top temperature. Since in the experiment, the side wall participated in the heat transfer process, the boundary conditions at this wall were not accurately known. For this reason, we carried out calculations for both perfectly conducting (D) and perfectly insulating (C) walls. In the vicinity of the cylinder's center $(r<0.3)$, there is little influence by the side wall, and the computations using either thermal boundary condition yielded similar results. The experimental data was also close to the computed results. As one approaches the side wall, the effect of the side wall thermal boundary condition becomes pronounced, and the 


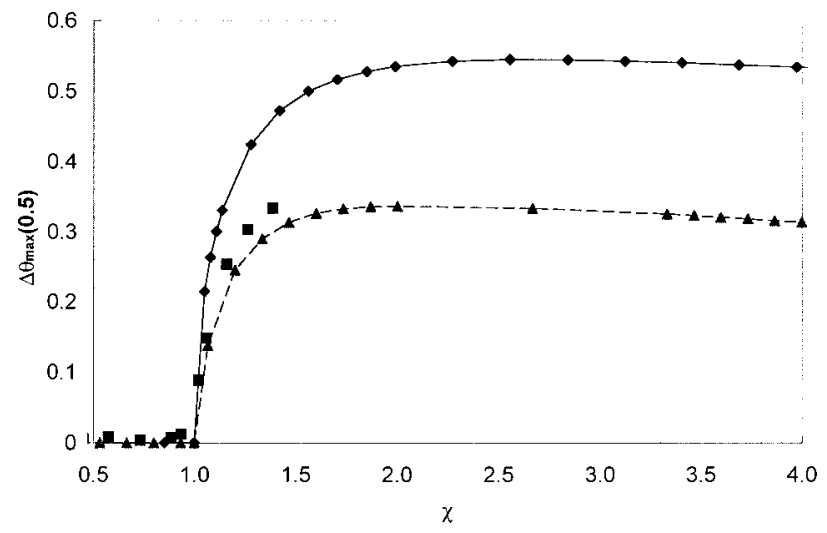

FIG. 7. The maximum mid-plane temperature difference, $\Delta \theta_{\max }(0.5)$, is depicted as a function of the reduced Rayleigh number $(\chi)$. Solid squares, solid diamonds, and solid triangles represent, respectively, experimental data, computational data for AC type thermal conditions, and computational data for AD type thermal conditions. $\operatorname{Pr}=135$ and $\gamma=0.5$.

computational results for the $\mathrm{C}$ and $\mathrm{D}$ type wall conditions deviated considerably. It is reassuring to note that the experimental data lies between the numerical results for insulating and perfectly conducting walls.

In the experiments, for low supercritical Rayleigh numbers, we used the difference between the maximum and minimum midplane temperatures,

$$
\Delta \theta_{\max }(0.5)=\max _{r, \varphi}(\theta(r, \varphi, 0.5))-\min _{r, \varphi}(\theta(r, \varphi, 0.5))
$$

as an indicator of the intensity of the convection. Figure 7 depicts $\Delta \theta_{\max }(0.5)$ as a function of the reduced Rayleigh number $(\chi)$. Solid squares, solid diamonds, and solid triangles represent, respectively, experimental data, computational data for $\mathrm{AC}$ type thermal conditions, and computational data for $\mathrm{AD}$ type thermal conditions. When $\chi<1$, in the absence of convection, $\Delta \theta_{\max }(0.5)=0$. When convection starts to gain importance $(1<\chi<1.5), \Delta \theta_{\max }(0.5)$ increases monotonically as a function of $\chi$. Again, the experimental data lies between the $\mathrm{AC}$ and $\mathrm{AD}$ cases. When $\chi$ is increased beyond $1.5, \Delta \theta_{\max }(0.5)$ initially varied little and eventually decreased slowly. For $\chi<1.5, \Delta \theta_{\max }(0.5)$ provides an indicator of the convection's intensity.

Thus far, we have described the flow and temperature fields in the cylinder in the absence of a controller. In the next section, we focus our attention on how a controller affects the flow patterns in the cylinder.

\section{THE CONTROLLED CASE}

In this section, we examine two types of controllers: a controller that controls the cylinder's bottom temperature distribution and a controller that controls the bottom's heat flux distribution. For example, the bottom of the experimental apparatus of Tang and $\mathrm{Bau}^{19}$ was equipped with 24 individually controlled heaters (actuators) mounted on a silicon wafer. The actuator's power supply was under computer control. Twenty-four diode sensors positioned at the cylinder's midheight continuously monitored the deviation of the midheight temperature distribution from the desired conductive

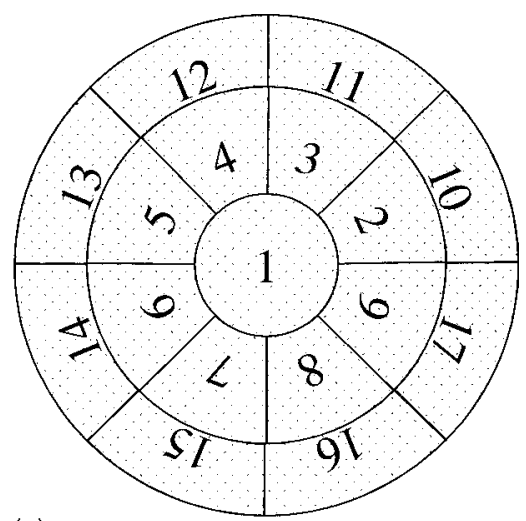

(a)

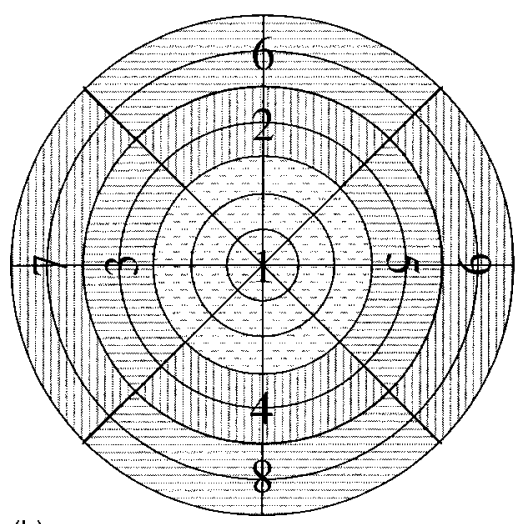

(b)

FIG. 8. The textured areas in (a) and (b) depict, respectively, 17 and 9 independently controlled actuators. When 56 actuators are used, each of the area elements acts as an independent actuator. The grid distribution on the heated surface is shown in Fig. 8(b).

values and directed the actuators to adjust their power supply in such a way as to counteract naturally occurring disturbances.

In order to economize in computer time, in most of the numerical simulations of the controller, we used a crude mesh of $7 \times 8 \times 7$ grid points. The investigations presented in Sec. III suggest that for the type of flow patterns existing in our cylinder, this grid provides qualitatively correct results.

In some of the numerical simulations, each of the area elements acted as an independent actuator to form a total of 56 actuators. In other numerical simulations, we combined a few of the elements [denoted by different textures in Figs. 8 (a) and 8(b)] to form 17 [Fig. 8(a)] and 9 [Fig. 8(b)] independently controlled actuators. Figure 8 (b) also shows the grid distribution on the heated surface. This corresponds to 56 actuators. The number of sensors matched exactly the number of actuators, and the sensors were located in the midheight plane above the actuators. In the numerical work, for computational convenience, we used actuators in the shape of circular segments. In contrast, in the experimental work, the actuators had a rectangular shape. ${ }^{19}$

In cases $\mathrm{A}$ and $\mathrm{B}$, the controller rules were specified, respectively, as

$$
\theta_{k}(z=0)=1-k_{T}\left(\theta_{k}(z=0.5)-0.5\right),
$$

and 


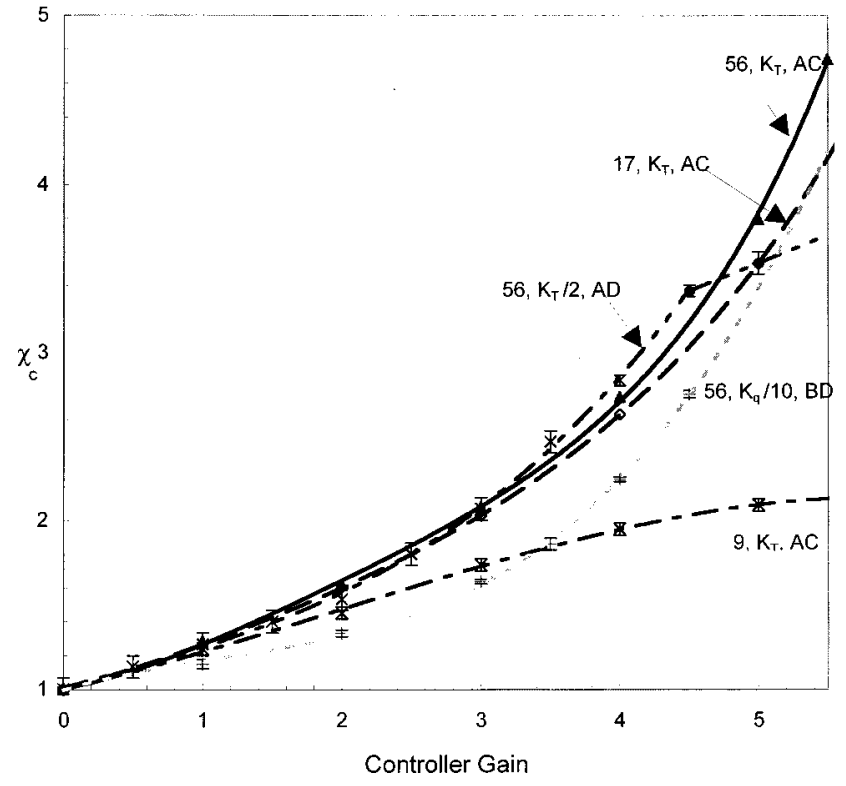

FIG. 9. The ratio between the critical Rayleigh numbers in the presence and the absence of the controller is depicted as a function of the controller gain for case $\mathrm{AD} 56$ actuators $\left(56, k_{T} / 2, \mathrm{AD}\right)$; case $\mathrm{AC}$ with $56\left(56, k_{T}, \mathrm{AC}\right), 17$ $\left(17, k_{T}, \mathrm{AC}\right)$ and $9\left(9, k_{T}, \mathrm{AC}\right)$ actuators; and case $\mathrm{BD}$ with 56 actuators $\left(56, k_{q} / 10, \mathrm{BD}\right) . \operatorname{Pr}=135$ and $\gamma=0.5$.

$$
\frac{\partial \theta_{k}(z=0)}{\partial z}=-1+k_{q}\left(\theta_{k}(z=0.5)-0.5\right),
$$

where the subscript $(k)$ identifies the sensors and the corresponding actuators. In the above, $k_{T}$ and $k_{q}$ are the proportional controller gains. Since the time constant of the convective processes in the fluid was on the order of minutes and the response time of the actuators was on the order of seconds, it was not necessary to account for time delays in Eqs. (2) and (3).

\section{A. Cases $A D$ and $A C$}

In order to study the effect of the controller on the critical Rayleigh number at the onset of convection, we specified the Rayleigh number and computed the flow field. Once the initial transient died out, we engaged the controller with various gains and observed whether the controller was able to successfully suppress convection. Case AD is investigated first.

For the AD case and 56 actuators, the curve denoted by $\left(56, k_{T} / 2, \mathrm{AD}\right)$ in Fig. 9 depicts $\chi_{c}$, the ratio of the critical Rayleigh numbers in the presence and absence of the controller as a function of the controller gain, $k_{T} / 2$. The symbol " $k_{T} / 2$ " indicates that the numerical values of the controller gain listed on the abscissa should be multiplied by a factor of 2 . In other words, the domain of the curve $\left(56, k_{T} / 2, \mathrm{AD}\right)$ in Fig. 9 is from 0 to 10 . Witness that as the controller gain increases, the critical Rayleigh number for the onset of convection increases as well. When $k_{T}<9$, the supercritical convection in the cylinder was qualitatively similar to that observed in the absence of the controller, and it predominantly consisted of a single convective cell. For larger controller gains $\left(k_{T}>9\right)$, the supercritical flow pattern in the controlled cylinder changed to predominantly axisymmetric convection.
This change of behavior was indicated by the abrupt change of slope of the $\chi_{c}\left(k_{T}\right)$ curve. As long as no flow existed, the bottom temperature was uniform and the isotherms were horizontal. The controller modified the stability characteristics of the no-motion (conductive) state without affecting the conductive state itself.

To illustrate the effect of the controller on the supercritical flow patterns, we depict in Fig. 10 the isotherms in the midplane $(z=0.5)$ when $R_{T}=29000, \operatorname{Pr}=135, k_{T}=0$ (a), $k_{T}=4$ (b), and $k_{T}=8.0$ (c). In the absence of the controller $\left[k_{T}=0\right.$, Fig. 10(a)], the flow consisted of a single cell; the flow ascended in one half of the cylinder and descended in the other half. When $k_{T}=4$ (b), the single cell flow structure was still in existence; but a larger fraction of the cross section was occupied by the ascending flow and a smaller fraction by the descending flow. When $k_{T}=8$ (c), the isotherms were consistent with axisymmetric flow. We emphasize that in Figs. 10(b) and 10(c), the bottom temperature was not uniform, and it was dictated by the controller rule [Eq. (2)]. The Rayleigh number was based on the nominal bottom temperature.

Since no experimental apparatus is perfect, of some concern was the ability of the controller to cope with imperfections. In order to assess the controller's ability to cope with imperfections, we specified a slightly non-uniform bottom temperature, i.e., we replaced the " 1 '" in Eq. (2) with $1+\varepsilon r \cos (\varphi)$. Under this condition, the purely conductive (no-motion) state no longer existed and weak convection was always present. When $\varepsilon=0.1$, Fig. 11 depicts the maximum midheight temperature difference, $\Delta \theta_{\max }(0.5)$, as a function of the reduced Rayleigh number $(\chi)$ and controller gains $k_{T}=0$ (solid diamonds), 3 (solid squares), and 4 (hollow triangles). As a result of the imperfection, the transition from the no-motion to the motion state is gradual and no longer abrupt as in the case of the perfect bifurcation (Fig. 7). The figure illustrates that even in the presence of imperfection, the controller still successfully reduces the intensity of the convective motion.

In the case $\mathrm{AC}$, smaller controller gains were needed to achieve the same relative increase in the critical Rayleigh number as in the AD case. This is perhaps due to the fact that the critical Rayleigh number for the onset of convection in case AD is about twice as large as in the AC case. When the number of heaters was 56, 17, and 9, the curves in Fig. 9 denoted by $\left(56, k_{T}, \mathrm{AC}\right),\left(17, k_{T}, \mathrm{AC}\right)$, and $\left(9, k_{T}, \mathrm{AC}\right)$ depict the reduced critical Rayleigh number $\left(\chi_{c}\right)$ as a function of the controller gain. In all these cases, as $k_{T}$ increases so does $\chi_{c}$. These curves illustrate the effect of the number of actuators on the controller's performance. In the range of parameters investigated here, the reduction in the number of actuators from 56 to 17 had little effect on the controller's performance. Further reduction to 9 actuators led, however, to a significant deterioration in the controller's performance. For example, when the controller's gain $k_{T}=5$, a control system with 9 and 56 actuators led, respectively, to a 2.1and a 4.8-fold increase in the critical Rayleigh number. In our experimental investigation, ${ }^{19}$ we used 24 actuators. The numerical simulations suggest that this is more than enough 


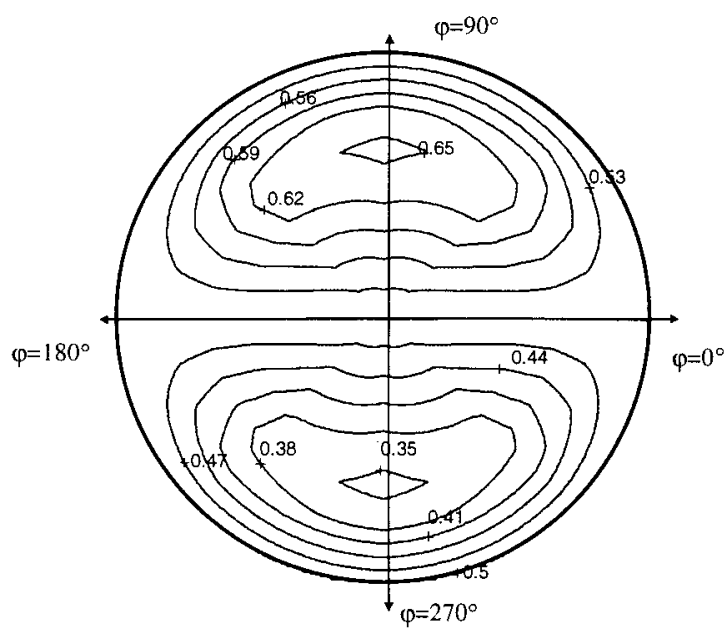

(a)

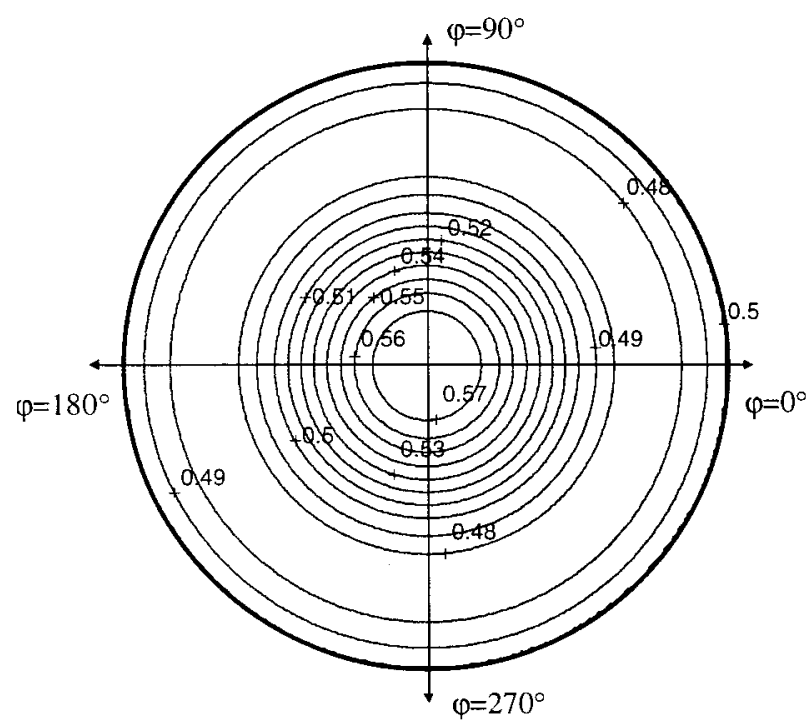

(c)

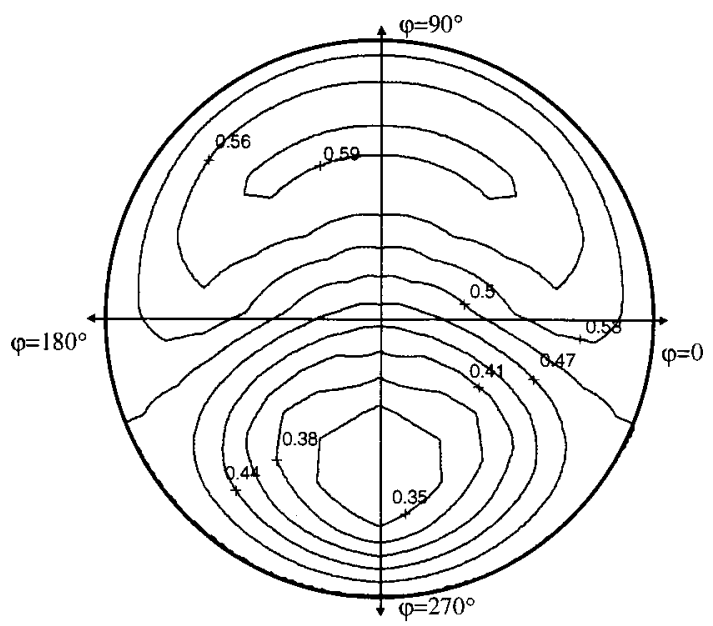

(b)

FIG. 10. The isotherms in the midplane $(z=0.5)$ are depicted when $R_{T}$ $=29000, \operatorname{Pr}=135, k_{T}=0$ (a), $k_{T}=4$ (b) and $k_{T}=8.0$ (c). Case AD. $\operatorname{Pr}$ $=135, \gamma=0.5$.

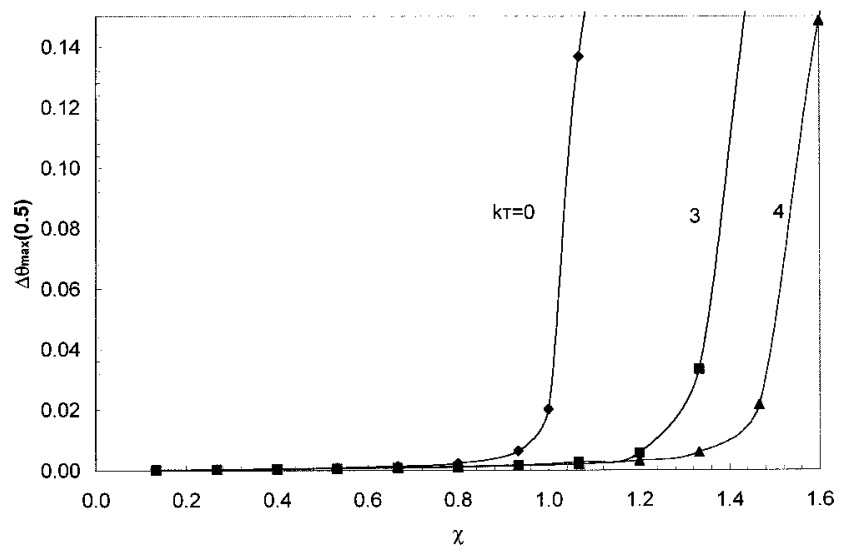

for effective control for the range of Rayleigh numbers considered in the experiment.

In order to identify other potential causes as to why the controller in the experiments did not perform as well as predicted by the theory, we investigate the effect of discontinuous changes in the actuator's output on the controlled system's performance. In the experiments, we used digital control that caused the actuator's output to vary by discrete jumps. To assess the effect of such changes on the controlled system's performance, we conducted a sequence of simulations in which we varied the actuators' output by finite increments corresponding to $10 \%$ of the nominal heated surface's temperature in the absence of a controller. In other words, we set the controller rule to be

When $\left(i-\frac{1}{2}\right) \times 0.1 \leqslant 1-k_{T}\left(\theta_{k}(0.5)-0.5\right)$

$$
\leqslant\left(i+\frac{1}{2}\right) \times 0.1 \text { then } \theta_{k}(0)=0.1 \times i \text {. }
$$

FIG. 11. In the presence of an imperfection, the difference between the maximum and minimum midplane temperatures is depicted as a function of the reduced Rayleigh number for various controller gains. Solid diamonds, solid squares, and hollow triangles correspond, respectively, to $k_{T}=0,3$, and 4. Case AD. $\operatorname{Pr}=135$ and $\gamma=0.5$. 

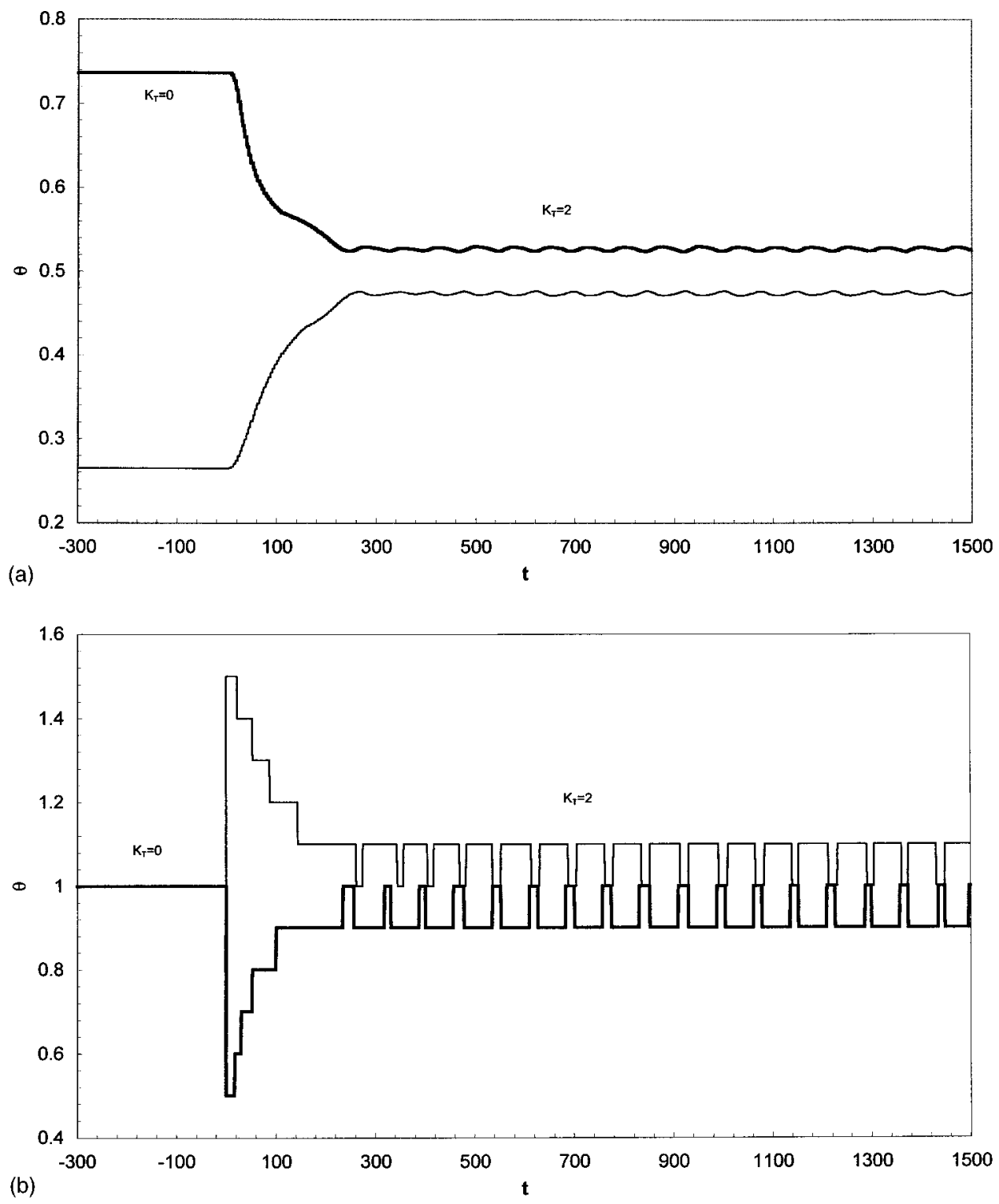

FIG. 12. The maximum and minimum midheight temperatures (a) and the corresponding actuators' output (b) are depicted as functions of time $(t)$. When $t<0$, the controller is off. The controller is applied at $t=0$ after steady convection has been established. Case AC. $R=5000, \chi=1.32$, the number of actuators is 17 , and the actuators' output is updated every 434 time steps by 0.1 increments. $\operatorname{Pr}=135$ and $\gamma=0.5$.

In the above, $(i)$ is a positive integer.

Figure 12 illustrates the effect of such a discrete controller on the system's performance. For case AC, Figs. 12(a) and 12(b) depict, respectively, the maximum and minimum midheight temperatures and the corresponding actuators' output as functions of time. In this simulation, $R=5000$, $\chi_{c}=1.32$, the number of actuators is 17 , and the actuators' output is updated every 434 time steps (which is equivalent to $\times 60 \mathrm{~s}$ ) by 0.1 increments. The dark (bottom) line in Fig. 12(b) corresponds to the dark (top) line in Fig. 12(a). At the start of the simulation, the controller is off, the actuators provide the nominal temperature (1) and convection develops in the cylinder. In the absence of control, the maximum difference in the cylinder's midheight temperatures is about 0.48 . Once steady convection has been established, the discrete controller is turned on at nondimensional time $t=0$. Initially, the turning on of the controller affects the actuators' temperature in a very significant way. However, as soon as the controller has reduced the intensity of the convection, the actuators' temperature returns to a value close to the nominal value of (1) and the midheight temperatures approach, but do not quite attain, the desired value of 0.5. When the controller is active, the maximum difference in the midheight temperature is about 0.06-an eightfold reduction from the uncontrolled case. Witness that the controller succeeds in suppressing established convection. This indicates that the controlled state has fairly sizable basin of attraction. Because of the discrete changes in the actuators' output, a time independent state is never established. The actuators' outputs oscillate around the nominal value of 1 , and the midheight temperatures undergo small amplitude oscillations. When the actuators' output is allowed to change continuously [Eqs. (1) and (2)] and in the absence of noise (not shown here), the midheight temperatures and actuators' output assume exactly the 

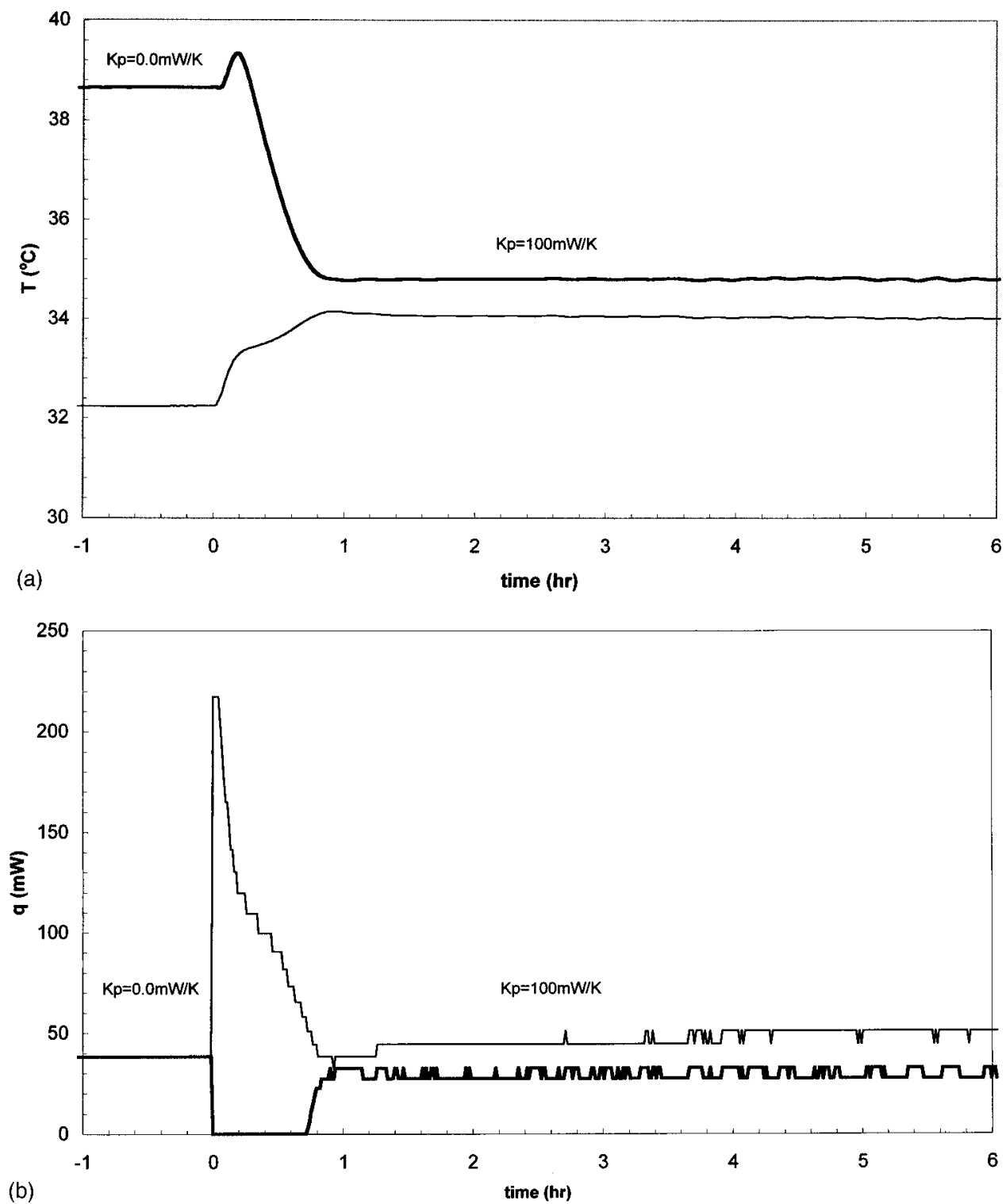

FIG. 13. The maximum and minimum temperatures $\left(\right.$ in ${ }^{\circ} \mathrm{C}$ ) recorded by diodes located at the cylinder's midheight (a) and the corresponding actuators' power variations $(\mathrm{mW})$ are depicted as functions of time. $\chi \sim 1.25$ and $k=100 \mathrm{~mW} / \mathrm{K}$. When $t<0$, the controller is off. The controller is turned on at $t=0$, after a steady convection has been established.

time-independent values of 0.5 and 1 , respectively, and a state of pure conduction is maintained.

The time series shown in Fig. 12 is interesting because it resembles closely our experimental observations. In Fig. 13, we reproduce a time series from our experimental data. ${ }^{19}$ Figures 13(a) and 13(b) depict, respectively, the maximum and minimum temperatures (in ${ }^{\circ} \mathrm{C}$ ) recorded by sensors located at the cylinder's midheight. $\chi_{c} \sim 1.25$ and $k=100 \mathrm{~mW} / \mathrm{K}$. Figure 13(b) depicts the power (in $\mathrm{mW}$ ) supplied to the actuators located directly beneath the diodes. When $t<0$, the controller was inactive and steady state convection was established inside the cylinder. At time, $t=0$, the controller was turned on. Similar to the theoretical investigation depicted in Fig. 12(b), the actuator reacts [Fig. 13(b)] by a sizable change in the actuator's signal. Once the controller successfully suppressed the convective motion, the actuator's signals return close to their nominal values and the mid- height temperatures return close to their conductive values [Fig. 13(a)]. In the absence of a controller, the maximum difference in midheight temperatures is about $\sim 6.2 \mathrm{~K}$. The controller reduces this temperature difference to less than 1 $\mathrm{K}$-about a sixfold decrease from the uncontrolled case. Similar to the numerical simulations, the controller in the experiment is robust and is able to suppress established motion. Because of the discrete changes in the actuators' output, a steady state is never established. The actuators' and sensors' signals fluctuate as functions of time. The amplitude of the temperature fluctuations [Fig. 13(a)] is very small and barely visible on the scale of the figure. The numerical simulations strongly suggest that the controller's inability in the experiment ${ }^{19}$ to reduce the difference between the maximum and minimum midheight temperatures to zero is due to discrete changes in the actuator's power which were necessitated by the limited resolution of the digital control system. 


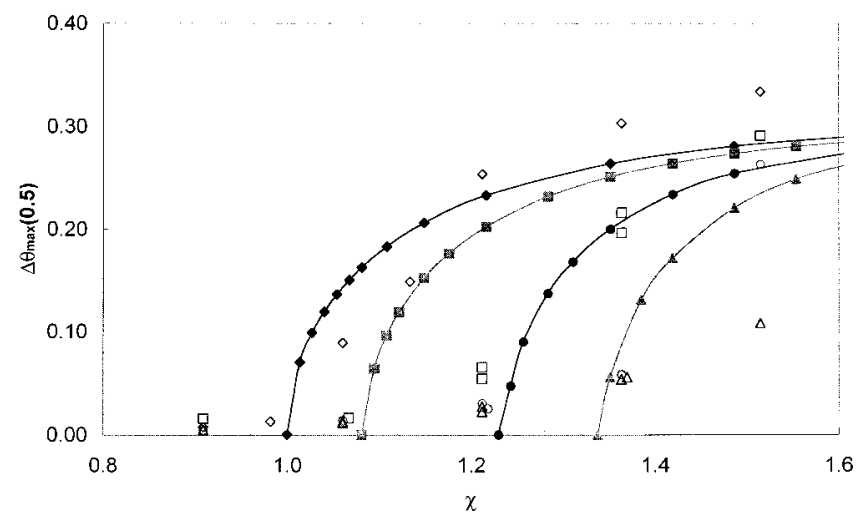

FIG. 14. The difference between the maximum and minimum midheight temperatures is depicted as a function of the reduced Rayleigh number for controller gains $k_{q}=0$ (solid diamonds), 2 (solid squares), 6 (solid circles), and 8 (solid triangles). The figure also includes experimental data depicted as hollow symbols. The hollow squares, hollow circles, and hollow triangles correspond, respectively, to controller gains $k_{e}, 2.2 k_{e}$, and $3.1 k_{e}$. Case BD. $\operatorname{Pr}=135$ and $\gamma=0.5$.

\section{B. Case BD}

Next, we describe the performance of the controller that modifies the bottom heat flux. The curve denoted $\left(56, k_{q} / 10\right.$, BD) in Fig. 9 depicts the reduced critical Rayleigh number $\left(\chi_{c}\right)$ as a function of the controller gain $\left(k_{q}\right)$. The " $k_{q} / 10$ ", implies that the values on the horizontal axis should be multiplied by 10 in order to get the controller gain. Similar to case A, the controller successfully retains a no motion state under conditions when cellular motion would have existed in the absence of the controller. For the range of controller gains investigated by us and for Rayleigh numbers slightly above critical, the motion was asymmetric and consisted of a single convective cell. For Rayleigh numbers much above criticality, axisymmetric motion was observed.

Figure 14 depicts the difference between the maximum and minimum mid-height temperatures as a function of the reduced Rayleigh number for controller gains $k_{q}=0$ (solid diamonds), 6 (solid squares), 15 (solid circles), and 20 (solid triangles). The figure also includes experimental data depicted as hollow symbols. Direct comparison between experiments and theory was complicated by the very high heat losses in our experimental apparatus which prevented us from obtaining an accurate relationship between the magnitude of the controller gain used in the experiment and the one used in the theoretical investigation. In Fig. 14, the hollow diamonds are the experimental data in the absence of the controller. The hollow squares, hollow circles, and hollow triangles correspond, respectively, to controller gains $k_{e}$, $2.2 k_{e}$, and $3.1 k_{e}$, where $k_{e}$ is the proportional controller gain in the experiment. The experimental data and the theoretical predictions exhibit similar trends. As the gain increases, the controller succeeds in maintaining a low midheight temperature difference for higher Rayleigh numbers. The discrepancies between theory and experiment can be attributed to the differences between the theoretical model and the experimental setup. We mention here only a few differences. In the experiment, imperfection made identification of the critical Rayleigh number difficult; discrete variations of

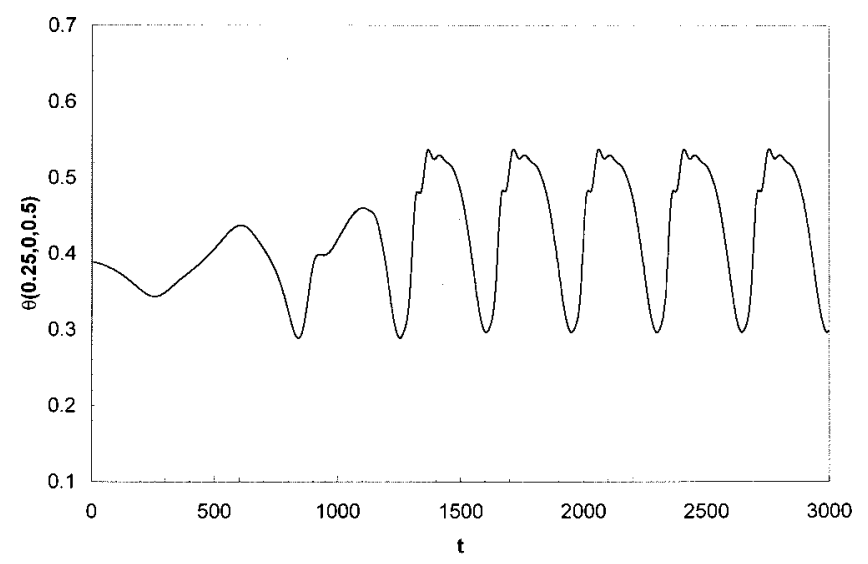

FIG. 15. The midheight temperature $\theta(0.25,0,0.5)$ is depicted as a function of time. Case BD. $\mathrm{Pr}=135, \gamma=0.5, k_{q}=60$, and $\mathrm{Ra}=30000$.

the actuators' power may have led to a deterioration in the controller's performance (see Figs. 12 and 13); temperature sensing at only a few spatial locations may have led to an underestimation of the maximal temperature difference; and participation of the side walls in the heat transfer process was more complex than what we assumed in the theoretical model.

At large controller gains (i.e., $k_{q}>50$ ), the bifurcation occurred directly from the no-motion state to time-periodic convection. For illustration purposes, Fig. 15 depicts the mid height temperature, $\theta\left(\frac{1}{4}, 0, \frac{1}{2}\right)$ as a function of time. $k_{q}=60$ and $\mathrm{Ra}=30000$. Witness that once initial transients have died out, the temperature oscillates as a periodic function of time. This oscillatory behavior is consistent with linear stability analysis of the no motion state of the controlled Rayleigh-Bénard convection in an infinite fluid layer. ${ }^{14}$ This analysis predicted that at high controller gains, a loss of stability of the no-motion state occurs through a Hopf bifurcation. In other words, at large controller gains, the "principle of exchange of stability" is no longer valid. It is interesting to note that as the fluid's Prandtl number increases, the Hopf bifurcation occurs at lower Rayleigh numbers. Similar oscillatory behavior was also observed in our experiments when large controller gains were used. ${ }^{11,19}$

The controller was fairly rugged, and it was able to counteract large disturbances. In some of our numerical simulations (i.e., Fig. 12), we started the numerical simulation with the controller being off. Once time-independent convection was established in the cylinder, we switched the controller on. The controller successfully suppressed the convective motion and established and retained a conductive, no motion state.

\section{CONCLUSIONS}

This paper describes a numerical investigation of Rayleigh-Bénard convection in an upright circular cylinder and the effect of a linear controller on the stability of the no-motion state. It was demonstrated that the no-motion state in a confined medium can be controlled and that the controller can counteract the adverse effects of imperfections. The controller is capable of suppressing established convection. 
The numerical code can be used to examine the performance of various control strategies. The numerical results are in qualitative agreement with experimental observations and with theoretical predictions for the unconfined case. Unfortunately, the degree of stabilization which was achieved in physical experiments falls short of theoretical predictions. The numerical simulations suggest that this shortcoming may have been caused by discrete variations in the actuators' power in the experiment. It is conceivable, therefore, that refinements in the experimental apparatus will improve the controller's capabilities and will bring its performance closer to that predicted by this theoretical work.

\section{APPENDIX: LINEAR STABILITY ANALYSIS}

In this Appendix, we use linear stability theory to compute the critical Rayleigh number for the onset of convection in an upright circular cylinder with uniform heat flux heating, an insulating side wall (case BC), nonslip velocity boundary conditions at the bottom and top boundaries, and a slip boundary condition at the side wall. This analysis is similar to the one carried out by Rosenblat ${ }^{33}$ for the onset of convection in a upright cylinder with a uniform bottom temperature. The results of these stability analyses were used to validate our numerical code.

Briefly, we linearized the Oberbeck-Boussinesq equations about the conductive, no motion state, $u=v=w=0$ and $\theta(r, \varphi, \zeta)=\frac{1}{2}-\zeta$, where $-\frac{1}{2} \leqslant \zeta \leqslant \frac{1}{2}$. After some manipulations, we obtained linear equations for the vertical component of the velocity, $w$, and for the deviation of the temperature, $\theta^{\prime}$, from the conductive temperature distribution.

$$
\operatorname{Pr}^{-1} \frac{\partial}{\partial t} \nabla^{2} w=R_{q} \nabla_{1}^{2} \theta+\nabla^{4} w
$$

and

$$
\frac{\partial \theta}{\partial t}=w+\nabla^{2} \theta
$$

where we dropped the superscript $\left({ }^{\prime}\right)$. The boundary conditions are

$$
\theta\left(r, \varphi, \frac{1}{2}\right)=\frac{\partial \theta\left(r, \varphi,-\frac{1}{2}\right)}{\partial z}=\frac{\partial \theta(\gamma, \varphi, z)}{\partial r}=0
$$

and

$$
w\left(r, \varphi, \pm \frac{1}{2}\right)=\frac{\partial w\left(r, \varphi, \pm \frac{1}{2}\right)}{\partial z}=\frac{\partial w(\gamma, \varphi, z)}{\partial r}=0 .
$$

In the above, $\nabla^{2}=\nabla_{1}^{2}+D^{2}, \quad \nabla_{1}^{2}=(1 / r)(\partial / \partial r)[r(\partial / \partial r)]$ $+\left(1 / r^{2}\right)\left(\partial^{2} / \partial \varphi^{2}\right)$, and $D=\partial / \partial z$. We proceed in the usual way by expanding $w$ and $\theta$ into the normal modes,

$$
\left(\begin{array}{c}
w \\
\theta
\end{array}\right)=\sum_{n, m, k}\left(\begin{array}{c}
W_{n, m, k} \\
\Theta_{n, m, k}
\end{array}\right)(z) f_{n, m, k}(r, \varphi) \exp \left(\sigma_{n, m, k} t\right),
$$

where $f_{n, m, k}(r, \varphi)$ satisfies the equation

$$
\nabla_{1}^{2} f+\lambda^{2} f=0 \text {. }
$$

$\lambda$ is a real positive number and the growth rate, $\sigma=\sigma_{R}$ $+i \sigma_{I} . \Theta$ satisfies the equation

$\left(\left(D^{2}-\lambda^{2}\right)\left(D^{2}-\lambda^{2}-\sigma\right)\left(D^{2}-\lambda^{2}-\operatorname{Pr}^{-1} \sigma\right)+\lambda^{2} R_{q}\right) \Theta=0$

with the boundary conditions

$$
\begin{aligned}
\Theta\left(\frac{1}{2}\right)=D \Theta\left(-\frac{1}{2}\right) & =\left(D^{2}-\lambda^{2}-\sigma\right) \Theta\left( \pm \frac{1}{2}\right) \\
& =D\left(D^{2}-\lambda^{2}-\sigma\right) \Theta\left( \pm \frac{1}{2}\right)=0 .
\end{aligned}
$$

Equation (A7) admits a solution of the form

$$
\Theta(z)=\sum_{i=1}^{3}\left(A_{i} \cosh \left(x_{i} z\right)+A_{i+3} \sinh \left(x_{i} z\right)\right),
$$

where $x_{i}$ 's are the roots of the polynomial equation

$$
\left(x^{2}-\lambda^{2}\right)\left(x^{2}-\lambda^{2}-\sigma\right)\left(x^{2}-\lambda^{2}-\operatorname{Pr}^{-1} \sigma\right)+\lambda^{2} R_{q}=0 .
$$

In order to have a nontrivial solution, we require that the determinant,

$$
\operatorname{Det}\left(\begin{array}{cc}
M & E \\
F & N
\end{array}\right)=0
$$

where

$$
\begin{aligned}
& M=\left(\begin{array}{ccc}
\cosh \left(\frac{x_{1}}{2}\right) & \cosh \left(\frac{x_{2}}{2}\right) & \cosh \left(\frac{x_{3}}{2}\right) \\
\Gamma_{1} \cosh \left(\frac{x_{1}}{2}\right) & \Gamma_{2} \cosh \left(\frac{x_{2}}{2}\right) & \Gamma_{3} \cosh \left(\frac{x_{3}}{2}\right) \\
\zeta_{1} \sinh \left(\frac{x_{1}}{2}\right) & \zeta_{2} \sinh \left(\frac{x_{2}}{2}\right) & \zeta_{3} \sinh \left(\frac{x_{3}}{2}\right)
\end{array}\right), \quad N=\left(\begin{array}{ccc}
x_{1} \sinh \left(\frac{x_{1}}{2}\right) & x_{2} \sinh \left(\frac{x_{2}}{2}\right) \quad x_{3} \sinh \left(\frac{x_{3}}{2}\right) \\
\Gamma_{1} \sinh \left(\frac{x_{1}}{2}\right) & \Gamma_{2} \sinh \left(\frac{x_{2}}{2}\right) & \Gamma_{3} \sinh \left(\frac{x_{3}}{2}\right) \\
\zeta_{1} \cosh \left(\frac{x_{1}}{2}\right) & \zeta_{2} \cosh \left(\frac{x_{2}}{2}\right) & \zeta_{3} \cosh \left(\frac{x_{3}}{2}\right)
\end{array}\right), \\
& E=\left(\begin{array}{ccc}
\sinh \left(\frac{x_{1}}{2}\right) & \sinh \left(\frac{x_{2}}{2}\right) & \sinh \left(\frac{x_{3}}{2}\right) \\
0 & 0 & 0 \\
0 & 0 & 0
\end{array}\right), F=\left(\begin{array}{ccc}
-x_{1} \sinh \left(\frac{x_{1}}{2}\right) & -x_{2} \sinh \left(\frac{x_{2}}{2}\right) & -x_{3} \sinh \left(\frac{x_{3}}{2}\right) \\
0 & 0 & 0
\end{array}\right)
\end{aligned}
$$




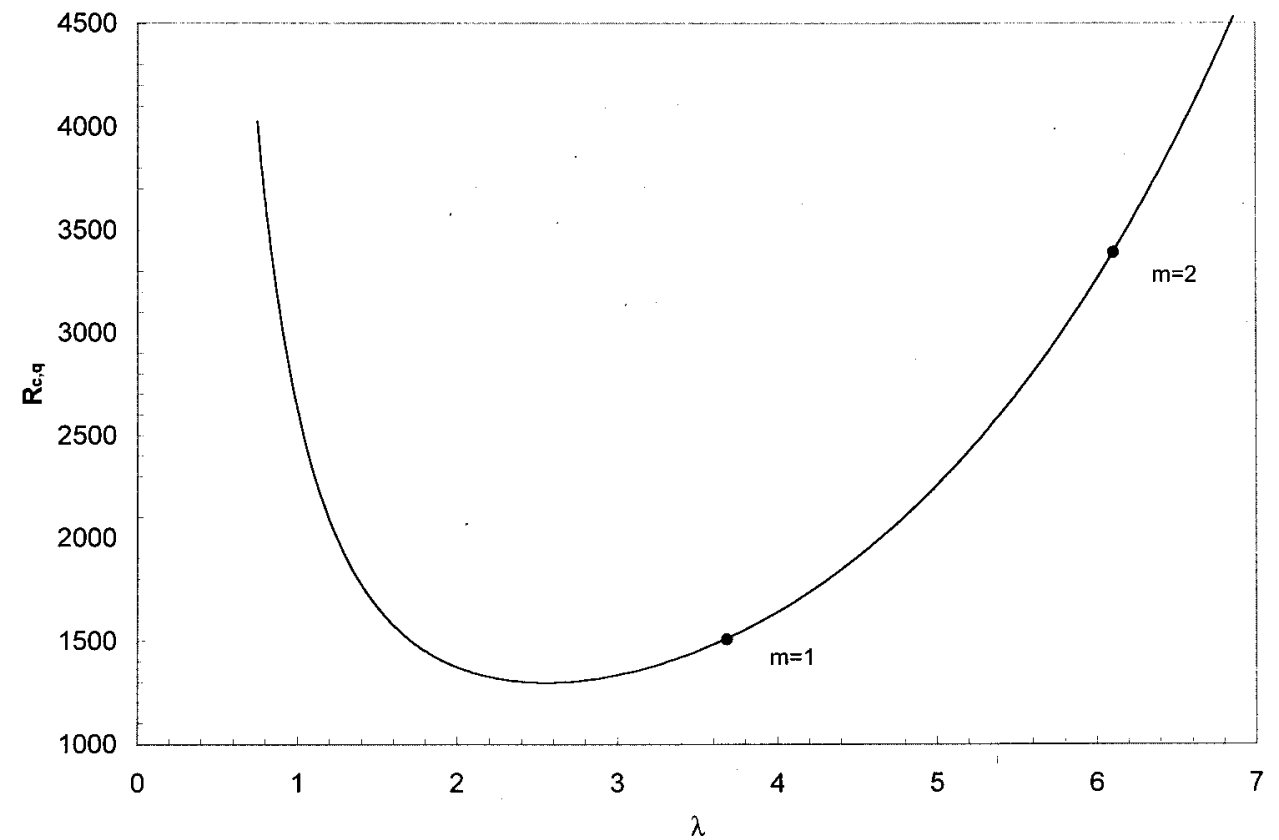

FIG. 16. The critical Rayleigh number is depicted as a function of the radial wave number, $\lambda$. The circles denote the admissible values of $\lambda$ when the azimutal wavenumber, $m$, equals 1 and 2 .

$\Gamma_{i}=x_{i}^{2}-\lambda^{2}-\sigma$, and $\zeta_{i}=x_{i} \Gamma_{i}$. To find the critical Rayleigh number, we set $\sigma_{R}=0$ in Eq. (A11). For a given value of $\lambda$, Eq. (A11) is an eigenvalue problem for $R_{q}$ and $\sigma_{I}$. In the absence of control, $\sigma_{I}$ is always zero and the critical Rayleigh number is independent of the Prandtl number. Figure 16 (solid line) depicts $R_{q}$ as a function of $\lambda$.

In the confined cylinder, $\lambda$ admits only discrete values. The solution of Eq. (A6) has the form, $f_{n, m}(r, \varphi)$ $=J_{m}\left(\lambda_{n} r\right) \exp (\operatorname{im} \varphi)$, where $m$ is an integer. The admissible values of $\lambda$ are determined by requiring that $(\partial / \partial r) J_{m}\left(\lambda_{n} \gamma\right)=0$. The first few values of $\lambda$ are $\lambda_{0} \sim 7.663, \lambda_{1} \sim 3.682$, and $\lambda_{2} \sim 6.108$. Accordingly, we have $R_{q 0} \sim 6122.6, R_{q 1} \sim 1508.4$, and $R_{q 2} \sim 3392.2$. The two lowest values of $R_{q}$ are denoted by circles in Fig. 16. The critical Rayleigh number is the smallest admissible one, $R_{q 1} \sim 1508.4$. This Rayleigh number corresponds to the azimutal wave number $m=1$, which corresponds to single cell, asymmetric supercritical convection and which is consistent with the results of our numerical computations as well as our experimental observations.

\section{ACKNOWLEDGMENTS}

This work was supported, in part, by Grant No. CTS9632237 from the National Science Foundation.

${ }^{1}$ J. Singer, Y.-Z. Wang, and H. H. Bau, "Controlling a chaotic system," Phys. Rev. Lett. 66, 1123 (1991).

${ }^{2}$ J. Singer and H. H. Bau, "Active control of convection," Phys. Fluids A 3, 2859 (1991).

${ }^{3}$ Y.-Z. Wang, J. Singer, and H. H. Bau, "Controlling chaos in a thermal convection loop," J. Fluid Mech. 237, 479 (1992).

${ }^{4} \mathrm{P}$. Yuen and H. H. Bau, "Rendering a subcritical bifurcation supercritical,", J. Fluid Mech. 317, 91 (1996).

${ }^{5} \mathrm{P}$. Yuen and H. H. Bau, "Controlling chaotic convection using neural nets-Theory and experiments," Neural Networks. 11, 557 (1988).
${ }^{6}$ S. H. Davis, "The stability of time periodic flows," Annu. Rev. Fluid Mech. 8, 57 (1976).

${ }^{7}$ R. J. Donnelly, "Externally modulated hydrodynamic systems," in Nonlinear Evolution of Spatio-Temporal Structures in Dissipative Continuous Systems, edited by F. H. Busse and L. Kramer (Plenum, New York, 1990), pp. 31-43.

${ }^{8}$ M. N. Roppo, S. H. Davis, and S. Rosenblat, "Bénard convection with time-periodic heating," Phys. Fluids 27, 796 (1984).

${ }^{9}$ R. E. Kelly, "Stabilization of Rayleigh-Bénard convection by means of a slow nonplanar oscillatory flow,' Phys. Fluids A 4, 647 (1992).

${ }^{10}$ R. E. Kelly and H. C. Hu, "The onset of Rayleigh-Bénard convection in a nonplanar oscillatory flow," J. Fluid Mech. 249, 373 (1993).

${ }^{11}$ J. Tang, "Active control of Rayleigh-Bénard convection," Ph.D. thesis, University of Pennsylvania, 1996.

${ }^{12}$ J. Tang and H. H. Bau, "Stabilization of the no-motion state in RayleighBénard convection through the use of feedback control,' Phys. Rev. Lett. 70, 1795 (1993).

${ }^{13}$ J. Tang and H. H. Bau, "Feedback control stabilization of the no-motion state of a fluid confined in a horizontal, porous layer heated from below," J. Fluid Mech. 257, 485 (1993).

${ }^{14}$ J. Tang and H. H. Bau, "Stabilization of the no-motion state in the Rayleigh-Bénard problem," Proc. R. Soc. London, Ser. A 447, 587 (1994).

${ }^{15}$ J. Tang and H. H. Bau, "Stabilization of the no-motion state of a horizontal fluid layer heated from below with Joule heating," Trans. ASME, J. Heat Transfer 117, 329 (1995).

${ }^{16}$ L. E. Howle, "Control of Rayleigh-Bénard convection in a small aspect ratio container,' 'Int. J. Heat Mass Transf. 40, 817 (1997).

${ }^{17}$ L. E. Howle, "Linear stability analysis of controlled Rayleigh-Bénard convection using shadowgraphic measurement," Phys. Fluids 9, 3111 (1997).

${ }^{18}$ L. E. Howle, “Active control of Rayleigh-Bénard convection," Phys. Fluids 9, 1861 (1997).

${ }^{19}$ J. Tang and H. H. Bau, "Experiments on the stabilization of the no-motion state of a fluid layer heated from below and cooled from above," J. Fluid Mech. 363, 153 (1998).

${ }^{20} \mathrm{H}$. Hu and H. H. Bau, "Feedback control to delay or advance liner loss of stability in planar Poiseuille flow," Proc. R. Soc. London, Ser. A 447, 299 (1994).

${ }^{21}$ H. H. Bau, "Control of Bénard-Marangoni convection," in review. 
${ }^{22}$ D. D. Joseph, Stability of Fluid Motions II (Springer-Verlag, Berlin, 1976).

${ }^{23}$ J. C. Buell and I. Catton, "The effect of wall conduction on the stability of a fluid in a right circular cylinder heated from below," Trans. ASME, J. Heat Transfer 195, 255 (1983).

${ }^{24}$ S. V. Patankar, Numerical Heat Transfer and Fluid Flow (McGraw Hill, New York, 1980).

${ }^{25}$ B. Leonard, "Stable and accurate convection modeling procedure based on quadratic upstream interpolation," Comput. Methods Appl. Mech. Eng. 19, 59 (1979).

${ }^{26}$ T. Hayase, J. A. C. Humphrey, and R. Greif, "A consistently formulated quick scheme for fast and stable convergence using finite volume iterative calculation procedure,'” J. Comput. Phys. 98, 108 (1992).

${ }^{27}$ Y. Saad, "Numerical solution of large nonsymmetric eigenvalue problems," Comput. Phys. Commun. 53, 71 (1989).
${ }^{28}$ C. A. Jones, D. R. Moore, and N. O. Weiss, “Axisymmetric convection in a cylinder,' J. Fluid Mech. 73, 353 (1976).

${ }^{29} \mathrm{G}$. Neumann, "Three-dimensional numerical simulation of buoyancy driven convection in vertical cylinders heated from below," J. Fluid Mech. 214, 559 (1990)

${ }^{30}$ G. S. Charlson and R. L. Sani, "'On thermoconvective instability in a bounded cyclindrical fluid layer," Int. J. Heat Mass Transf. 13, 1479 (1971).

${ }^{31}$ G. Muller, G. Neumann, and W. Webber, "Natural convection in vertical Bridgman configurations,' J. Cryst. Growth 70, 78 (1984).

${ }^{32}$ S. Schneider and J. Straub, "Laminar natural convection in cylindical enclosure with different end temperatures," Int. J. Heat Mass Transf. 35, 545 (1992).

${ }^{33}$ S. Rosenblat, "Thermal convection in a vertical circular cylinder," J. Fluid Mech. 122, 395 (1982). 